\title{
Larval fish dispersal in a coral-reef seascape
}

\author{
Glenn R. Almany ${ }^{1 \dagger}$, Serge Planes ${ }^{1}$, Simon R. Thorrold ${ }^{2 \star} \oplus$, Michael L. Berumen ${ }^{3} \oplus$, Michael Bode ${ }^{4}$, \\ Pablo Saenz-Agudelo ${ }^{1,3,5}$, Mary C. Bonin ${ }^{6}$, Ashley J. Frisch ${ }^{6,7}$, Hugo B. Harrison ${ }^{6}$, Vanessa Messmer ${ }^{6}$, \\ Gerrit B. Nanninga ${ }^{3,8}$, Mark A. Priest ${ }^{3,9}$, Maya Srinivasan ${ }^{6}$, Tane Sinclair-Taylor ${ }^{3}$, David H. Williamson ${ }^{6}$ \\ and Geoffrey P. Jones ${ }^{6}$
}

\begin{abstract}
Larval dispersal is a critical yet enigmatic process in the persistence and productivity of marine metapopulations. Empirical data on larval dispersal remain scarce, hindering the use of spatial management tools in efforts to sustain ocean biodiversity and fisheries. Here we document dispersal among subpopulations of clownfish (Amphiprion percula) and butterflyfish (Chaetodon vagabundus) from eight sites across a large seascape $\left(10,000 \mathrm{~km}^{2}\right)$ in Papua New Guinea across 2 years. Dispersal of clownfish was consistent between years, with mean observed dispersal distances of $15 \mathrm{~km}$ and $10 \mathrm{~km}$ in 2009 and 2011 , respectively. A Laplacian statistical distribution (the dispersal kernel) predicted a mean dispersal distance of 13-19 km, with $90 \%$ of settlement occurring within 31-43 km. Mean dispersal distances were considerably greater (43-64 km) for butterflyfish, with kernels declining only gradually from spawning locations. We demonstrate that dispersal can be measured on spatial scales sufficient to inform the design of and test the performance of marine reserve networks.
\end{abstract}

R obust descriptions of larval dispersal are fundamental to studies of fish population dynamics ${ }^{1,2}$, fisheries management ${ }^{3,4}$ and the design of reserve networks tasked with conserving ocean biodiversity ${ }^{5,6}$. Yet descriptions of larval dispersal patterns in ocean environments remain scarce. The combination of a pelagic larval phase that may last several days to many months and an ocean environment characterized by energetic diffusive and advective flows may allow passive larvae to disperse hundreds to thousands of kilometres from natal locations $s^{7,8}$. It has proved difficult, however, to verify directly how far fish larvae travel, because it is almost impossible to follow them as they disperse rapidly from spawning sites and are subject to high rates of natural mortality throughout the larval phase $e^{9}$. Our inability to describe the spatial extent of larval dispersal is problematic because our understanding of metapopulation dynamics relies on largely untested models that quantify where larvae arriving at a subpopulation originate from and where larvae spawned at each subpopulation eventually settle ${ }^{10-12}$. Moreover, to be of practical use, these data must be assembled on large enough scales for evaluating and optimizing spatial management strategies for fisheries or conservation ${ }^{1,13}$.

Patches of reef habitat are frequently isolated from each other by deeper water that forms a barrier to adult movement, and so larval dispersal is likely to be a critical process in the persistence of many reef fish populations over demographic and evolutionary timescales ${ }^{10,14}$. Effective management of coral-reef seascapes is therefore particularly reliant on spatial tools to achieve conservation objectives. Although reef fish larvae clearly have the potential for long-distance movements ${ }^{14}$, there is increasing evidence that dispersal may be more limited than previously assumed ${ }^{15,16}$. The most compelling evidence of larvae returning to natal or nearby reefs has come from chemical labelling of embryos ${ }^{17,18}$ and genetic DNA parentage analyses ${ }^{19-27}$. However, few studies have been able to fully describe a dispersal kernel by determining the distances over which spatially fragmented subpopulations are connected by larval movements. Here we combine a comprehensive, large-scale genetic parentage study with a new method of fitting dispersal kernels to describe patterns of dispersal for orange clownfish (Amphiprion percula) and vagabond butterflyfish (Chaetodon vagabundus) among marine reserves across a large coral-reef seascape $\left(\sim 10,000 \mathrm{~km}^{2}\right)$. Orange clownfish lay demersal eggs, with embryos hatching after 5 days and larvae spending 10-12 days in the pelagic environment ${ }^{18}$. Vagabond butterflyfish spawn pelagic eggs that hatch in less than 24 hours and then spend $28-45$ days as pelagic larvae. We have previously established that a significant proportion of juveniles recruit to natal reefs for populations of both species at Kimbe Island, Papua New Guinea $(\mathrm{PNG})^{18,22}$, but bidirectional movement patterns of larvae across the Kimbe Bay seascape remain unknown. Effective conservation strategies are vital, as members of both fish families are targeted by the aquarium fish $\operatorname{trade}^{28}$ and are susceptible to local extirpation as a result of habitat degradation caused by the development of coastal land ${ }^{29}$. An understanding of larval connectivity is critical to ensure that reserve networks are designed to maximize the probability of population persistence in the face of rapid environmental changes ${ }^{18}$.

\section{Results}

We undertook intensive field sampling in 2009 and 2011 to quantify larval dispersal in the two study species on a scale that encompassed a network of locally managed marine areas throughout Kimbe Bay, on the north coast of New Britain Island in PNG. Seven of the eight

\footnotetext{
'Laboratoire d'Excellence CORAIL EPHE, PSL Research University, UPVD, CNRS, USR 3278 CRIOBE, BP 1013, 98729 Papetoai, Moorea, French Polynesia. ${ }^{2}$ Biology Department, Woods Hole Oceanographic Institution, Woods Hole, Massachusetts 02543, USA. ${ }^{3}$ Red Sea Research Center, Division of Biological and Environmental Science and Engineering, King Abdullah University of Science and Technology, Thuwal, 23955, Saudi Arabia. ${ }^{4}$ ARC Centre of Excellence for Environmental Decisions, School of BioSciences, University of Melbourne, Parkville, Melbourne, Victoria 3010, Australia. ${ }^{5}$ nstituto de Ciencias Ambientales y Evolutivas, Universidad Austral de Chile, Chile. ${ }^{6}$ ARC Centre of Excellence for Coral Reef Studies, and College of Science and Engineering, James Cook University, Townsville, Queensland 4811, Australia. ${ }^{7}$ Reef HQ, Great Barrier Reef Marine Park Authority, Townsville, Queensland 4810, Australia. ${ }^{8}$ Department of Zoology, University of Cambridge, Downing Street, Cambridge CB2 3EJ, UK. ${ }^{9}$ Marine Spatial Ecology Lab, School of Biological Sciences, University of Queensland, Queensland 4072, Australia. 'Deceased. *e-mail: sthorrold@whoi.edu
} 
$150^{\circ} \mathrm{W}$
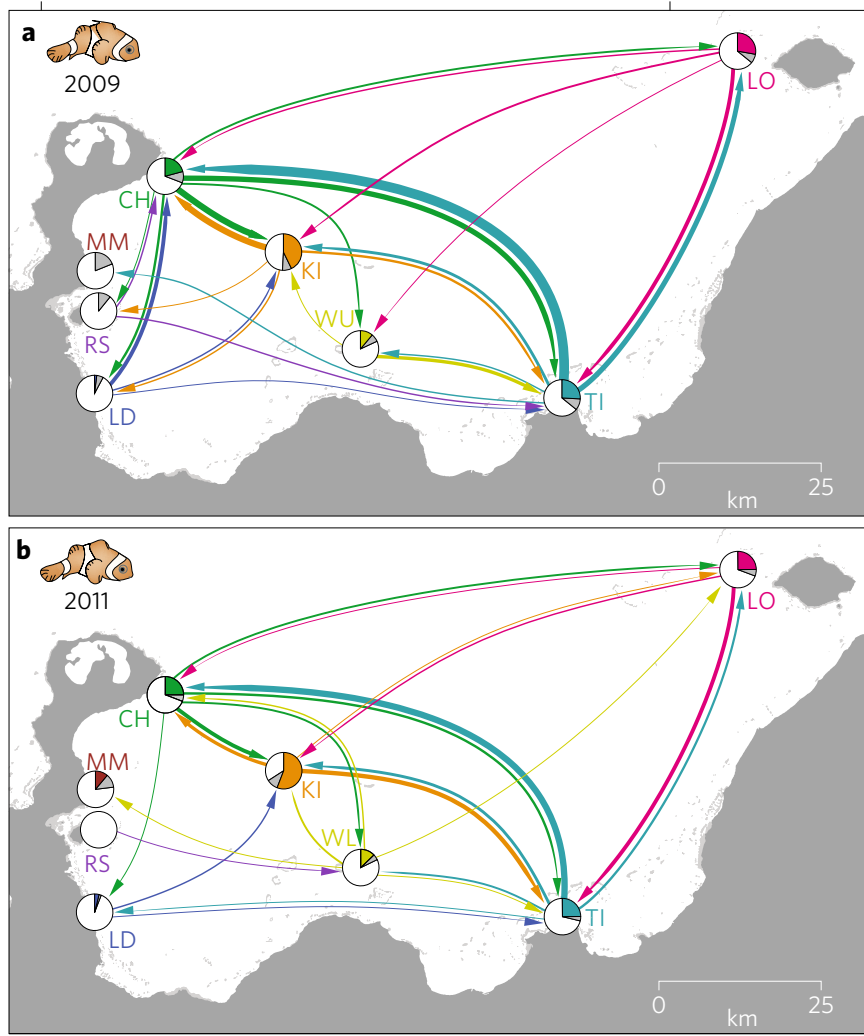

$150^{\circ} \mathrm{W}$
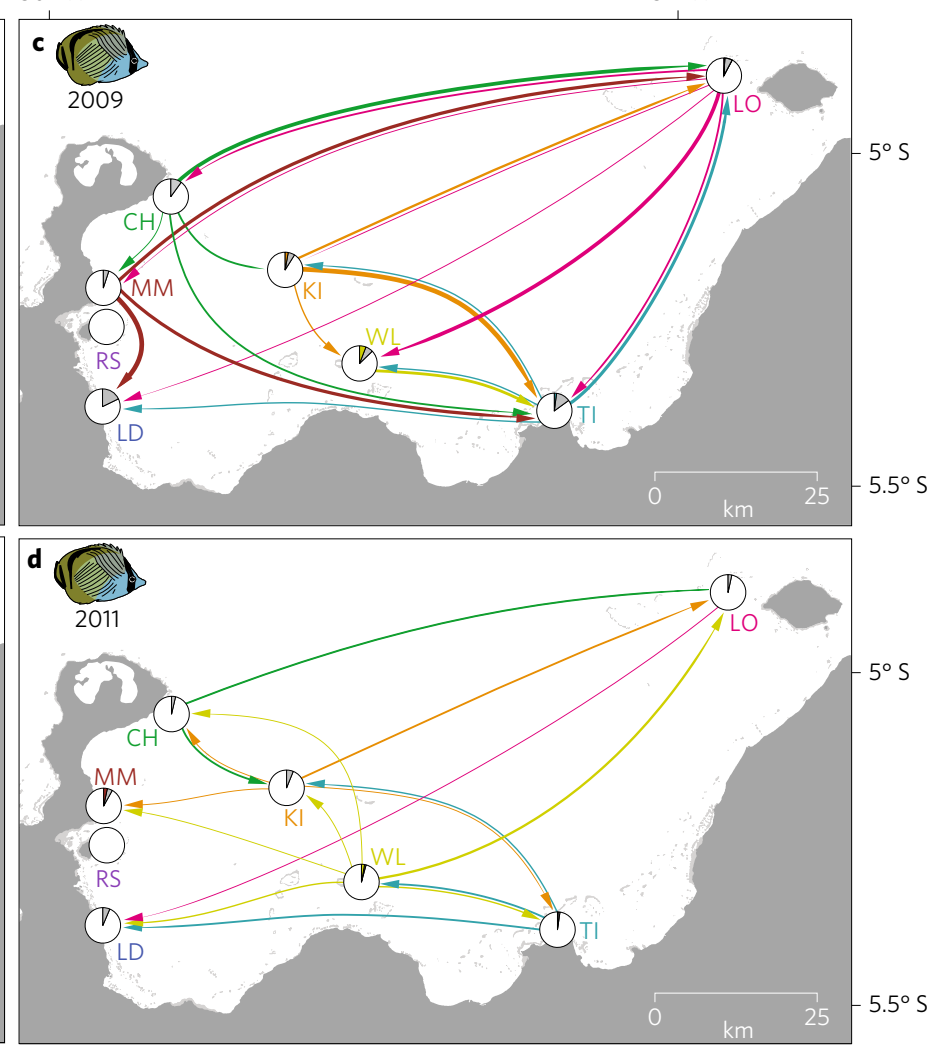

Figure 1 | Network diagram of larval dispersal of two reef fish species among study sites within Kimbe Bay, Papua New Guinea. a-d, Adult and juvenile Amphiprion percula $(\mathbf{a}, \mathbf{b})$ and Chaetodon vagabundus (c,d) were collected from eight reef areas including Walindi (LD), Restoff/Shumann (RS), Malu Malu Islands (MM), Cape Huessner (CH), Kimbe Island (KI), Wulai Islands (WU), Tairobe (TI) and Lolobau (LO) in 2009 and 2011. Pie charts show the proportion of newly settled juveniles collected that were spawned at the same location (coloured segments), proportion of juveniles collected that were spawned at one of the other study locations (grey segments) and proportion of juveniles that were not assigned to any adult in the study (white segments). Thickness of connecting lines is proportional to the number of individuals that dispersed between sites (see Supplementary Table 1 for raw data).

sites included fringing reefs associated with small islands, where the two focal species were particularly abundant. These sites were all designated as marine protected areas in the management plan for Kimbe Bay designed by the Nature Conservancy ${ }^{30}$ and are managed on a local scale by the traditional reef owners in nearby villages. The other location (Walindi reefs) was a small group of fringing reefs near the Mahonia $\mathrm{Na}$ Dari Research and Conservation Center, some of which have been designated under PNG fisheries legislation as no-take marine reserves ${ }^{30}$. Pair-wise distance among locations varied from $10 \mathrm{~km}$ to $120 \mathrm{~km}$. At each of the eight sites, reef area varied in size from 0.42 to $1.36 \mathrm{~km}^{2}$, and the total area of reef habitat throughout Kimbe Bay was $94 \mathrm{~km}^{2}$.

We collected fin-clips from almost all adult pairs of $A$. percula and a significant proportion (ranging from 12\% to 77\%) of adult C. vagabundus populations at each of the eight sites in both years (Supplementary Table 1). A total of 2,546 adult A. percula and 2,021 adult C. vagabundus were sampled in 2009, and 2,913 adult $A$. percula and 4,858 adult C. vagabundus were sampled in 2011. We also collected juveniles of both species that had settled into reef habitats within 6 months of the adult collections. A total of 1,447 juvenile A. percula and 985 juvenile C. vagabundus were collected in 2009, and 1,547 juvenile $A$. percula and 958 juvenile C. vagabundus were collected in 2011. We genotyped all A. percula samples at 15 (for 2009) and 22 (for 2011) microsatellite DNA loci, and C. vagabundus samples at 15 (for 2009) and 19 (for 2011) loci (Supplementary Table 3). The resulting DNA fingerprints were used to match juveniles with potential parents in a log-likelihood statistical framework ${ }^{31}$. We assigned 407 (28.1\% of those sampled) juvenile A. percula independently to both parents in the same anemone in 2009, and similarly $437(28.2 \%)$ juveniles were assigned in 2011. For C. vagabundus, 53 juveniles (5.4\% of those sampled) were assigned to one or both parents in 2009, and 36 (3.8\%) juveniles were assigned in 2011 (Supplementary Table 1). The resulting matrix of larval dispersal distances from natal reefs (observed dispersal) was then used to test models of dispersal kernels that accounted for differences in population size, incomplete sampling of adults and reefs, the presence of unassigned juveniles and the distribution of reef habitat throughout the seascape. Kernels were constructed for both species and in both years by matching several probability density functions to the observed dispersal matrices (see Supplementary Information).

Both $A$. percula and C. vagabundus exhibited a high degree of connectivity, with exchange of larvae not only between neighbouring reserves (10-30 km apart) but also among reefs up to $120 \mathrm{~km}$ apart, in both 2009 and 2011 (Fig. 1). For A. percula, exchange among sub-populations was noticeably more frequent among sites supporting the largest sub-populations $(\mathrm{CH}, \mathrm{KI}$ and $\mathrm{TI})$ than those with smaller numbers of adults (Fig. 1a,b; Supplementary Table 1). In contrast, we observed no obvious pattern in connectivity among sites as a function of population size for C. vagabundus (Fig. 1c,d), albeit with lower numbers of parental assignments compared with A. percula (Fig. 1c,d). Levels of self-recruitment were considerably higher for $A$. percula in both years (mean $=12.9 \%$ in 2009 and $20.2 \%$ in 2011$)$ than for C. vagabundus $(0.3 \%$ in 2009 and $0.01 \%$ in 2011). Self-recruitment in A. percula was consistently highest at Kimbe Island (KI; Fig. 1), an isolated offshore island, and lowest at coastal sites within Kimbe Bay. 


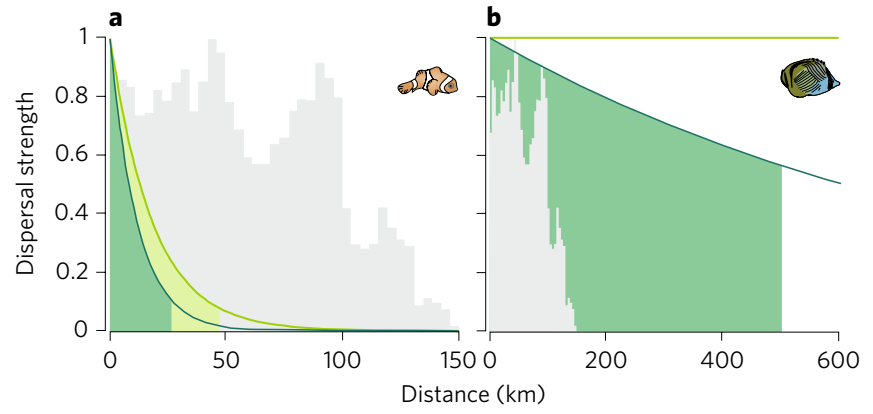

Figure 2 | Larval dispersal kernels for two reef fish species from Kimbe Bay, Papua New Guinea. a,b, Expected dispersal kernels (solid lines) were fitted using an exponential decay function to observed connectivity measured in A. percula (a) and C. vagabundus (b) from collections in 2009 (light green lines and shaded areas) and 2011 (dark green lines and shaded areas). Shaded areas indicate distance at which $90 \%$ of the larvae of each species are expected to settle, while grey bars indicate the relative amount of suitable habitat available at each inter-reef distance throughout the study area. None of the models converged for C. vagabundus data in 2009.

The shapes of dispersal kernels estimated for the two focal species were markedly different and probably reflected, to some degree, respective differences in pelagic larval durations and dispersal potential. Connectivity declined rapidly as a function of source distance in 2009 and 2011 for A. percula (Fig. 2a). Mean observed dispersal distances for $A$. percula were $15.0 \mathrm{~km}$ (95\% confidence intervals (CI): 8.4-23.8) in 2009 and $10.0 \mathrm{~km}$ (CI: 8.1-13.6) in 2011. Most larvae were also dispersing over shorter distances than predicted based on the geographical spacing of reefs within Kimbe Bay (Fig. 2). The observed distances were best fitted by an exponentially declining kernel in both years, with a mean modelled dispersal distance of $18.9 \mathrm{~km}$ (95\% CI: $13.4-25.4$ ) in 2009 and $13.3 \mathrm{~km}$ (95\% CI: 11.1-19.1) in 2011 (Supplementary Fig. 1). Based on these probability density functions, $90 \%$ of $A$. percula juveniles settled within $43 \mathrm{~km}$ of their parents in 2009 , compared with $31 \mathrm{~km}$ in 2011. We also fitted separate dispersal kernels for each of the three zones within Kimbe Bay in 2009 and 2011 (Supplementary Fig. 2). Confidence intervals (95\%) for the individual kernels overlapped with the combined kernel in both years, suggesting that there were no significant spatial patterns in dispersal within Kimbe Bay. These results suggest that Kimbe Bay represents a largely closed metapopulation for the clownfish. Unassigned juveniles in the samples from the eight sites presumably either came from outside the boundaries of the study or, more likely, from other unsampled adults within Kimbe Bay.

The derived dispersal kernel estimated for the butterflyfish, C. vagabundus, predicted considerably longer dispersal than for A. percula, with no discernable decline in settlement rates over distances of up to $150 \mathrm{~km}$ in 2009 and only a gradual decline in 2011 (Fig. 2b). An exponential model produced the best fit for observed dispersal in 2011, whereas no function could be fitted to the 2009 data. The mean observed dispersal distance was $63.8 \mathrm{~km}$ (95\% CI: 47.7-73.6) in 2009 and $43.3 \mathrm{~km}$ (95\% CI: $35.2-55.7)$ in 2011. However, the exponential model predicted a mean dispersal distance for $2011(220 \mathrm{~km})$ that was much greater than the mean observed dispersal distances and, based on bootstrapped 95\% confidence intervals, the function could not be distinguished from a flat distribution (Supplementary Fig. 1). Together, these results suggest that, despite an extensive sampling effort, we were unable to sample the tail of the dispersal kernel adequately for C. vagabundus. Individuals on Kimbe Bay reefs are presumably part of a large population that is connected along the north coast of New Britain and beyond.



$\square<0.0001 \square \geq 0.0001,<0.001 \square \geq 0.001,<0.05 \square \geq 0.05$



Figure 3 | Larval connectivity matrices for A. percula in Kimbe Bay. a-e, We calculated origin $(\mathbf{a}, \mathbf{c})$ and arrival $(\mathbf{b}, \mathbf{d})$ connectivity matrices among all reefs within west, mid and east Kimbe Bay (e) based on larval dispersal kernels from 2009 (a,b) and 2011 (c,d). The location of each anemone is identified by a coloured dot indicating its assigned zone (west, blue; mid, cyan; east, green). Diagonal elements in origin matrices represent local retention (the fraction of offspring produced at a site that recruits to that same site), whereas diagonals in arrival matrices represent self-recruitment (the fraction of recruitment to a site comprising individuals spawned at that same site) $)^{13}$.

Descriptions of the full dispersal kernel for A. percula enabled us to calculate connectivity matrices based on both larval origin (the fraction of larvae dispersing to each location from each location) and arrival (the fraction of juveniles found on a given reef that have come from each location) in 2009 and 2011 (Fig. 3). Both metrics are necessary to provide a complete picture of self-replenishment and connectivity ${ }^{10-12}$, but have not previously been estimated from empirical data. The two connectivity metrics were surprisingly similar, with strong diagonal elements indicating high local retention for the origin matrices and high self-recruitment for the arrival matrices. Local retention in this instance was defined as the number of larvae that left reef $\mathrm{X}$ and survived and settled on reef $\mathrm{X}$ as a proportion of the number of larvae that left reef $X$ and survived and settled somewhere else. Similarly, self-recruitment was defined as the number of larvae from reef $\mathrm{X}$ that arrived at reef $\mathrm{X}$ as a proportion of the total number of larvae arriving at reef X. Both local retention and self-recruitment were also higher in 2011 than in 2009. The open water separating the eastern and western sides of Kimbe Bay clearly represented a significant barrier to clownfish connectivity, 
with almost all connections between the east and west groups of reefs being mediated through reefs in the centre of the bay. The central coastal sites were important nodes of connectivity that served to effectively link the reefs in the bay into a single metapopulation. Patterns of connectivity were, however, heterogeneous across Kimbe Bay. Western and central reef locations were well connected to each other, but less well connected to sites in eastern Kimbe Bay. Our results demonstrate that it is possible to make individual parentage assignments across large distances within a coral-reef seascape. These observations can, in turn, be used to estimate parameters for spatially explicit population models in order to inform design criteria for reserve networks and provide a method for evaluating the degree to which reserves may export larvae to fished areas outside their boundaries ${ }^{23}$.

\section{Discussion}

Coral-reef seascapes are under increasing pressure from global, regional and local effects linked to human activities ${ }^{32}$. Reef systems with spatially explicit management regimes are thought to increase resilience to anthropogenic stressors ${ }^{3}$. Yet significant knowledge gaps and limited availability of empirical measurements preclude the development of optimal strategies for the design of marine reserve networks and other spatial management tools that can be generalized from individual species to reef fish communities. Our results are in broad agreement with two studies on coral groupers and snappers from the Great Barrier Reef ${ }^{24}$ and Manus Island ${ }^{26}$ that identified dispersal events up to $30 \mathrm{~km}$ away from spawning locations. However, a recent study ${ }^{25}$ found that a pelagic-spawning goby inhabiting sponges on the Belize barrier reef with a larval duration of 18-34 days had a median dispersal distance of only $1.8 \mathrm{~km}$, an order of magnitude lower than our estimates for A. percula, which spawn demersal eggs and have a much shorter pelagic larval duration (PLD). Marine reserves would need to be placed considerably closer together than is commonly achieved if the results from gobies in Belize are the rule rather than the exception and dispersal distances are commonly less than $10 \mathrm{~km}$ in coral-reef fishes. In contrast, a Red Sea clownfish with a PLD of less than 2 weeks was recently shown to exhibit virtually no self-recruitment to natal reefs ${ }^{33}$, highlighting the risk of assuming that small marine protected areas will be able to experience enough self-recruitment to sustain local populations ${ }^{34}$. Median dispersal distances of $100-200 \mathrm{~km}$ were recently reported for two species of coral groupers with average PLDs of 26 days in the southern Great Barrier Reef ${ }^{35}$, highlighting the apparent lack of a predictive relation between larval duration and dispersal distance in coral-reef fishes. It is unfortunate that PLD is not a better proxy for dispersal distances in coral-reef fishes, as it is significantly quicker and cheaper to apply than the DNA parentage approach used in all the above studies. However, a recent study ${ }^{36}$ found that a more conventional (and logistically less challenging) approach based on a genetic isolation-by-distance model was able to accurately estimate mean larval dispersal distance in the same A. percula populations that were sampled in the present study. This result provides some hope that future empirical estimates of larval dispersal in coral-reef fishes may be routinely achieved using the isolation-by-distance method rather than relying on DNA parentage analyses.

Larvae of coral-reef fish are regularly dispersing tens of kilometres throughout the Kimbe Bay seascape, but the physical and biological processes generating the observed patterns remain unresolved. The physical environment of Kimbe Bay is characterized by high-relief bathymetry with numerous reefs and islands that influence current flows and potentially impact the larval dispersal patterns of reef fishes. Tides are diurnal with a maximum amplitude of approximately $1 \mathrm{~m}$ in the Bismarck Sea, indicating that tidal currents are relatively sluggish throughout the region. Mesoscale eddies are present in Kimbe Bay, at least some of which are hypothesized to originate from instabilities in the South Equatorial Current and
New Guinea Coastal Current ${ }^{37}$. Mesoscale eddy activity may have been responsible for the widely varying levels of self-recruitment of C. vagabundus at Kimbe Island over a 7-year period. Observed rates of self-recruitment were much higher in $2005(\sim 60 \%)$ than in 2007, 2009 or 2011, for which measured rates in each of the three years were $<5 \%$. Eddies have been hypothesized to play important roles in the successful return of larval coral-reef fishes to benthic habitats ${ }^{38}$ and in the survival of reef fish larvae ${ }^{39}$. It is possible that mesoscale eddies are similarly influencing connectivity patterns in Kimbe Bay. However, the often unpredictable and ephemeral nature of mesoscale eddies means that it will be difficult to quantify their effects without considerable effort to fully understand the hydrodynamic environment within Kimbe Bay.

Larval dispersal of reef fishes is likely to be influenced by the behavioural abilities of larvae along with ambient current flows. Most larval reef fishes are strong swimmers with well-developed sensory systems that together may allow more limited dispersal than would be predicted based on advection and dispersion of passive particles ${ }^{40}$. Recently settled $A$. percula juveniles can detect olfactory signals in waters treated with anemones and leaves from rainforest trees, and can distinguish between water collected at locations adjacent to reefs and offshore water ${ }^{41}$. This ability may, in turn, allow clownfish larvae to orientate in the water column so as to avoid movements away from natal reefs, or alternatively to better locate suitable settlement habitat at the end of the pelagic larval phase. Although DNA parentage analysis provides a spawning and settlement location for each juvenile that is genetically assigned to its parents, potential differences in individual larval trajectories cannot be identified from this approach. Otolith geochemistry profiles of $A$. percula larvae settling on their natal reef on Kimbe Island were indistinguishable from those of larvae that had arrived from other locations, suggesting that both larval dispersal types inhabited water with similar physical characteristics ${ }^{42}$. Tracking reef fish larvae in the open ocean remains a challenge that will probably require the development of new approaches before more progress can be made.

Our findings add to a growing literature that suggests networks of marine reserves can function both to help conserve biodiversity ${ }^{43}$ and as an effective tool for fisheries management ${ }^{44}$. Yet few studies have assessed the relative importance of processes that potentially shape dispersal kernels in the ocean ${ }^{45}$. Such information is critical to the development of a mechanistic understanding of larval connectivity in reef seascapes because larval dispersal and the design of marine protected-area networks probably interact to determine the persistence of reef fish populations ${ }^{46}$. Although marine reserves alone are not a sufficient hedge against many of the threats facing coral reefs ${ }^{47}$, they are likely to continue to be used as a primary tool for ocean conservation and sustainable fisheries by much of the developing world. Our study is a necessary step towards developing predictive models and algorithms to optimize the design of marine reserve networks and attain the full benefits of integrated spatial management. We demonstrate that it is possible to empirically measure dispersal kernels of coral-reef fishes over spatial and temporal scales that are relevant to the design and performance evaluation of marine reserve networks. Without such data, it is not possible to test predictive models of dispersal used for the design of reserve networks. Ultimately, the performance of these management actions is likely to determine whether the ecosystem functions and services provided by coral reefs are preserved in the face of continued exploitation of reef biota and the increasing impacts of climate change.

\section{Methods}

Work was carried out under ethics approval A1643 from James Cook University, research visas approved by the PNG government, and research protocols endorsed by the Board of Mahonia Na Dari Research and Conservation Centre, Kimbe, PNG.

We studied the dispersal dynamics of two species of coral-reef fishes with differing life-history strategies in Kimbe Bay, which is centrally located on the north coast of the island of New Britain in PNG. The clown anemonefish or orange 
clownfish (Amphiprion percula) lays demersal eggs, with embryos hatching after 5 days and larvae spending 10-12 days in the pelagic environment ${ }^{18}$. Vagabond butterflyfish (Chaetodon vagabundus) spawn pelagic eggs that hatch in less than 24 hours and then spend $28-45$ days as pelagic larvae $e^{17}$. Both species are abundant on the reef flats ( $<1 \mathrm{~m}$ depth) and lagoons $(<10 \mathrm{~m}$ depth) of coastal fringing reefs and offshore islets in Kimbe Bay.

Sampling design and sample size. Adults of our two study species were sampled over 4 weeks in April 2009 and 4 weeks in March 2011. The clownfish, A. percula, was sampled by divers, using scuba and snorkel, who searched all suitable habitat and mapped the locations of all anemones on reefs using GPS. Collectors captured all adult pairs with hand nets using a mixture of clove oil and ethanol as an anaesthetic when necessary. Individuals were measured, and small pieces of fin tissue were taken from the caudal fin and preserved in $85 \%$ ethanol in individual $2.0-\mathrm{ml}$ vials. All handling of the fish was carried out underwater, next to the anemone, with individuals returned to their home anemones in less than 2 minutes.

Adults of the vagabond butterflyfish, C. vagabundus, were captured by using barrier nets, hand nets, clove oil and micro-spears in six of the eight areas (Walindi reefs and Restoff/Schumann Islands were not sampled, owing to local political restrictions and low numbers of adult fish). Adults were fin-clipped in situ and released. The conspicuous fin clip, consistently taken from the same location on the dorsal fin of each sampled individual, ensured that the same fish were not re-sampled during the study period.

Juveniles of our two study species were sampled concurrently with the adult sampling in April 2009 and in March 2011. For A. percula, all anemones that were sampled for adults were also sampled for new recruits (individuals $<2.5 \mathrm{~cm}$ ). All new recruits were captured using hand nets and measured using calipers, and then killed and preserved whole in $85 \%$ ethanol.

For C. vagabundus, snorkellers searched all suitable inshore habitat for juveniles. Juveniles were captured using hand nets and clove oil, were measured using calipers and were preserved in $85 \%$ ethanol. Juvenile C. vagabundus were collected from all eight areas.

A total of 17,275 individuals of both species were sampled for parentage analysis in 2009 and 2011. This included 2,546 adult and 1,447 juvenile A. percula that were sampled in 2009, and 2,913 adult and 1,547 juveniles in 2011 (Supplementary Table 1). For C. vagabundus, 2,021 adults and 985 juveniles were sampled in 2009 and 4,858 adults and 958 juveniles in 2011 (Supplementary Table 1).

Genetic techniques and parentage analysis. Genomic DNA was extracted from $\sim 2 \mathrm{~mm}^{2}$ of fin tissue from each sample by using the Qiagen DNeasy blood and tissue kit, following the manufacturer's protocol. Microsatellite loci were amplified using the Type-it Microsatellite PCR kit (Qiagen) following the manufacturer's protocol with annealing temperatures between 57 and $63^{\circ} \mathrm{C}$. Primers were fluorescently labelled and pooled in multiple multiplex reactions with up to five loci per reaction. Microsatellite panels, multiplex groups, number of alleles, PCR product range and original reference describing each microsatellite used in this study are indicated in Supplementary Table 2. All PCR products were screened on an ABI 3730XL automated sequencer (Applied Biosystems). Allele sizes were determined with the fragment analysis software GENEMAPPER 4.0. All loci for both species satisfied Hardy-Weinberg and linkage equilibrium assumptions.

Amphiprion percula. For 2009, at least 12 of the 15 microsatellite loci in the panel (Supplementary Table 1) were successfully amplified for 3,993 individuals (2,546 adults and 1,447 juveniles). For 2011, a total of 4,492 individuals (2,913 adults and 1,579 juveniles) were successfully amplified by PCR for at least 19 of the 22 loci in the panel (Supplementary Table 2). Missing data were distributed among all loci and accounted for less than $2 \%$.

Chaetodon vagabundus. In 2009, 3006 individuals (2,021 adults and 985 juveniles) were successfully amplified by PCR for at least 12 of the 15 loci in the panel (Supplementary Table 1). Missing data accounted for less than $1 \%$ and were distributed among all loci. In 2011, 5,816 individuals (4,858 adults and 958 juveniles) were successfully amplified by PCR for at least 16 of the 19 loci in the panel (Supplementary Table 1). Missing data accounted for less than $2 \%$ and were distributed among all loci.

Categorical allocation of parent-offspring relationships was assessed based on a maximum likelihood approach implemented in the software program $\mathrm{Famoz}^{28}$. The cumulative exclusion probabilities for the microsatellite panel used in each data set are detailed in Supplementary Table 3. The program computes log of the odds ratio (LOD) scores for parent-offspring relationships and constructs statistical tests for parentage assignments. These tests are based on the simulation of offspring from genotyped parents (true pairs) and from allele frequencies estimated from the genetic data set (false pairs) to construct statistical tests for parentage assignments. In the present study, 10,000 simulated offspring were generated from genotyped parents and allele frequencies for each data set. These simulations allow the inclusion of an error term to take into account genotyping error. We used an error rate of $0.01 \%$ for $C$. vagabundus data sets and $0.001 \%$ for $A$. percula data sets that minimized statistical errors associated with parentage tests ${ }^{48}$. Minimum LOD score thresholds for accepting single-parent and two-parent assignments as being true were defined as the intersection between the two distributions of LOD scores from simulated offspring (true versus false pairs) mentioned previously (Supplementary Table 3). This parameter set was evaluated using the 'parentage test simulation' option to estimate the probability of excluding a true parent, knowing that it was in the sample (type I error), and the probability of assigning a false parent, knowing that the true parent was not sampled (type II error).

All juveniles were screened against the total pool of adult samples to identify parent-offspring relationships. For all data sets, missing data accounted for less than $5 \%$ and were distributed across all loci. As a conservative measure to reduce possible false assignments, we excluded from further analyses all single-parent assignments that had LOD scores higher than the assigned threshold but presented more than two mismatches between the genotype of the offspring and the genotype of the assigned parents. Mean LOD scores $( \pm 1$ s.d.) for accepted assigned pairs in each data set are shown in Supplementary Table 3.

Estimating population abundance. It was necessary to estimate population abundance in order to estimate the proportion of the adult population sampled and compare the magnitude of percentage self-recruitment and observed pair-wise connectivity among locations. For A. percula, $>95 \%$ of all adult individuals were sampled from all sites except for Wulai and Tairobe. For these two sites, total population sizes were estimated from the proportion of the total area sampled and the total population size estimate for the area sampled (Supplementary Table 4). This figure was used to convert the observed proportion of juveniles assigned to parents to an estimate of self-recruitment (\%) for these sites (Supplementary Table 4) following the method described elsewhere ${ }^{24}$. It was also used to provide a population-weighted estimate of the relative connectivity among populations.

Population sizes of adult C. vagabundus were estimated at the six sites where adults were sampled, using visual transects and a stratified random sampling design $^{49}$. A total of $\sim 3,700$ visual transects were undertaken across the six locations before the adult sampling, with transects distributed among all habitat types. Ground-truth surveys of habitat types were made by snorkellers, and the habitats were subsequently digitized using ArcGIS 9.2 (ESRI) at a scale of 1:4,000 using 1-m-resolution satellite imagery (IKONOS) to estimate the area of each habitat. Snorkellers surveyed each habitat type using a towed GPS unit that recorded the length of each transect and the positions at which individual C. vagabundus were observed. Densities within each strata were calculated, and a total population estimate was derived from an estimate of the area of each strata calculated using ArcGIS (Supplementary Table 5), with greater sampling effort placed in larger and more variable habitats ${ }^{22,49}$. Given a known number of adult fish that were finclipped, we were able to calculate the overall percentage of the population that was sampled for genetic analysis. This figure was used to convert the observed proportion of juveniles assigned to parents to an estimate of percentage selfrecruitment for each location (Supplementary Table 5). It was also used to provide a population-weighted estimate of the relative connectivity among populations.

Fitting dispersal kernels accounting for unsampled adults. Reef locations were estimated by applying ArcGIS 9.2 (ESRI) to the digitized map of Kimbe Bay. A total of 888 separate reefs were identified, and the area of each was calculated from its vector outlines. All Euclidean distances between them were calculated, based on the calculated reef centroid. Using this information, dispersal kernels were then fitted to the empirical data using a new method that takes into account both sampled and unsampled reefs, and adjusts for differences in population size among reefs.

The method assumed that the metapopulation is made up of $P$ patch populations, each with a population of $N_{i}$ individuals (this could be either the number of adults, given an equal sex ratio, or the number of females). Adult sampling took place on a subset $\mathbf{S}_{\mathrm{A}}$ of these patches, which had $s_{\mathrm{A}}$ elements. Sampling of post-recruitment juveniles took place on a subset $\mathbf{S}_{\mathrm{J}}$, which had $s_{\mathrm{J}}$ elements. The method does not assume that all adults and juveniles were sampled on any reef, although more complete sampling of either will yield better statistical power. We therefore denote $\xi_{i}$ as the proportion of adults sampled on reef $i$, with $\xi_{i}=0$ for those reefs that were completely unsampled (the majority). Adult and juvenile sampling occurred at the same time, and so the two sampled subsets often contain the same reefs.

Each of the sampled juveniles had its parentage assessed, and either was assigned to a sampled adult or had its parentage classified as unknown. This count data populates an $\left(\left(s_{\mathrm{A}}+1\right) \times s_{\mathrm{J}}\right)$ matrix denoted $\mathbf{M}$, with the columns indicating the reef to which the juveniles recruited. The rows indicate the reef containing the parents. The first $s_{\mathrm{A}}$ rows contain reefs in the sampled set, and the final row pools all juveniles from unknown parents (Supplementary Table 6). Depending on the relative number, size and spatial distribution of sampled and unsampled adult populations, this final row can dominate the matrix.

These count data are essentially a sample of each reef's recruits, which are themselves samples from the settlement pool. We assume that this pool is very large compared with the number of successful settlers, and therefore that we can model recruitment as a sample with replacement from the pool. A large settlement pool also allows us to ignore sampling effects in the composition of the pool itself. 
We finally also assume that each larva, regardless of its origin, has an equal probability of settling successfully (that is, there is no local adaptation).

We assume that the settlement pool (and thus the parentage data that are sampled from it) is a realization of a dispersal process that is isotropic, distancebased and spatially invariant. Larvae are released from the natal reef $i$, which has a centroid located at $\left(x_{i}, y_{i}\right)$. After the dispersal process has occurred, the density of larvae at a sampled reef $j$, whose centroid is located at $\left(x_{j}, y_{j}\right)$ in space, is entirely determined by the Euclidean distance between the locations:

$$
d_{i j}=\sqrt{\left(x_{i}-x_{j}\right)^{2}+\left(y_{i}-y_{j}\right)^{2}}
$$

The dispersal kernel is a distance-based probability density function $\rho(k, d)$. We constructed a likelihood function that compares the predictions of given kernel functional forms and parameter values to the parentage matrix. One common dispersal kernel is the generalized Gaussian:

$$
\rho(k, d)=\exp \left[-(k d)^{m}\right]
$$

where the constant $m$ defines the kernel shape. The classical exponential decay model, also known as the Laplacian, is given where $m=1 ; m=2$ gives the Gaussian distribution (a bell curve) and $m=3$ gives a Ribbens function ${ }^{26}$. Normally such kernels have standardizing coefficients to ensure that - as probability density functions - they integrate to 1 . However, because we were interested in the proportional composition of the settlement pool, any such coefficients would not affect the kernel fit (see equation (3)) and so we did not include them. The process was to use a particular kernel $\rho(d)$ and parameter set $k$ to model the proportional composition of the settlement pool at each reef where juveniles were sampled. The proportion of the larvae in the settlement pool of reef $j$ that come from sampled adults on reef $i$ was:

$$
q_{i j}(k)=\frac{\left[1-\left(1-\xi_{i}\right)^{2}\right] N_{i} \rho\left(k, d_{i j}\right)}{\sum_{m=1}^{P} N_{m} \rho\left(k, d_{m j}\right)}
$$

The numerator of this equation corresponds to the number of larvae that were spawned by sampled adults at reef $i$ and that disperse into the settlement pool of reef $j$. The denominator normalizes this number by dividing the size of the total settlement pool on reef $j$. Both the numerator and denominator would be modified by the per capita fecundity of the females, the proportion of successfully fertilized eggs and the mortality during the larval dispersal phase. However, we assumed that these parameters do not vary between the different reefs, and therefore the parameters do not alter model fits. As an example, if our dispersal kernels were Gaussian with shape parameter $k$, the proportional composition of individuals from the sampled population on reef $i$ in the settlement pool of reef $j$ would be:

$$
q_{i j}(k)=\frac{\left[1-\left(1-\xi_{i}\right)^{2}\right] N_{i} \exp \left[-\left(k d_{i j}\right)^{2}\right]}{\sum_{m=1}^{P} N_{m} \exp \left[-\left(k d_{m j}\right)^{2}\right]}
$$

These equations are partly defined by the population size on the potential source reefs. This will often be unknown; an estimate of $N_{j}$ can be based on the average density of comparable reef habitat on sampled reefs. Ideally, the uncertainty in these density estimates will be propagated through to alter the uncertainty of the kernel estimates. Although we did not apply this variation to our dispersal kernel fits, they would ideally be included by using repeated fits, in which the density on unknown reefs in each case was chosen at random from observed variation.

We assumed that the observed recruits at reef $j$ were a sample with replacement from the settlement pool, and therefore from the probabilities given in equation (3). Not all reefs in the system were sampled, and we therefore found that a large proportion of juveniles in the sample could not be attributed to any particular reef. The chosen dispersal kernel also indicates that the proportion of the settlement pool at reef $j$ comprising unattributed individuals from both unsampled and partially sampled reefs will be:

$$
\bar{q}_{j}(k)=\frac{\sum_{i=1}^{P}\left(1-\xi_{i}\right)^{2} N_{i} \rho\left(k, d_{i j}\right)}{\sum_{m=1}^{P} N_{m} \rho\left(k, d_{m j}\right)}
$$

The log-likelihood of observing the set of attributed and unattributed samples contained in the parentage matrix $\mathbf{M}$, given a kernel defined by parameter set $k$, is thus:

$$
\mathrm{LL}(k \mid \mathbf{M})=\sum_{j=1}^{P} \ln \left(\begin{array}{c}
R_{j} \\
\mathbf{M}_{i j}
\end{array}\right)+\mathbf{M}_{X j} \ln \left(\bar{q}_{j}(k)\right)+\sum_{i \in \mathbf{S}_{\mathbf{A}}} \mathbf{M}_{i j} \ln \left(q_{i j}(k)\right)
$$

where the total number of recruits sampled on reef $j$ is denoted $R_{j}$. The index variable $X$ in $\mathbf{M}_{X j}$ refers to the final row in the parentage matrix, which contains the numbers of unallocated juveniles on each reef. Note that the presence of the unattributed juvenile samples in the fitting process was essential to securing a precise and accurate picture of the dispersal kernel shape.

We generated confidence intervals around the best-fit parameter by bootstrap resampling the data, using the source reefs as resampling units. That is, we resampled the columns of each row in the parentage matrix with replacement.

Modelling dispersal for A. percula and C. vagabundus in Kimbe Bay. Based on the matrix of inter-reef distances, dispersal kernels were extrapolated to estimate the best-fit dispersal kernels for each species, in each year. We considered three candidate single-parameter dispersal kernels with exponential form (Laplacian, Gaussian, and Ribbens; Supplementary Table 6). According to maximum likelihood, the Laplacian kernel fitted the data best for all A. percula data sets, and the Ribbens kernel fitted the C. vagabundus data set best for 2011. In 2009, none of the candidate kernels was able to effectively recreate the C. vagabundus data set.

Based on the matrix of inter-reef distances, dispersal kernels can be extrapolated to estimate the relative amount of dispersal $N_{i} \rho\left(d_{i j}\right)$ travelling from reef $i$ to reef $j$ in the metapopulation. We constructed two connectivity matrices that describe two different aspects of dispersal among the reefs in Kimbe Bay - the proportion of larvae that departed each reef for other reefs in the metapopulation (origin matrix), and the proportional composition of larvae that arrived on each reef (arrival matrix). Specifically, we define an arrival matrix $\mathbf{A}$, whose elements $A_{i j}$ denote the proportion of recruits on reef $j$ whose parents are from reef $i$. The diagonal elements $A_{i i}$ of this matrix therefore measure self-recruitment. We also define an origin matrix $\mathbf{E}$, whose elements $E_{i j}$ denote the proportion of settling larvae from reef $i$ (larvae that settled on a reef somewhere in the bay) who land on reef $j$. The diagonal elements $E_{i i}$ of this matrix are the proportional local retention, a quantity closely related to the absolute local retention. The matrices are defined as:

$$
\begin{gathered}
A_{i j}=\frac{N_{i} \rho\left(k, d_{i j}\right)}{\sum_{m=1}^{P} N_{m} \rho\left(k, d_{m j}\right)} \\
E_{i j}=\frac{\rho\left(k, d_{i j}\right)}{\sum_{m=1}^{P} \rho\left(k, d_{i m}\right)}
\end{gathered}
$$

These matrices differ in both the denominator and the inclusion of the population sizes in the calculation.

Data availability. All data supporting the findings of this study are available within the paper and its Supplementary Information. Microsatellite primer sequences are provided in Supplementary Table 2, along with original reference describing the microsatellites used in this study.

\section{Received 20 July 2016; accepted 22 March 2017; published 8 May 2017}

\section{References}

1. Botsford, L. W. et al. Connectivity, sustainability, and yield: bridging the gap between conventional fisheries management and marine protected areas. Rev. Fish. Biol. Fish. 19, 69-95 (2009).

2. Kritzer, J. \& Sale, P. F. Metapopulation ecology in the sea: from Levin's model to marine ecology and fisheries science. Fish Fisheries 5, 131-140 (2004).

3. Gell, F. R. \& Roberts, C. M. Benefits beyond boundaries: the fishery effects of marine reserves. Trends Ecol. Evol. 18, 448-455 (2003).

4. Sale, P. F. et al. Critical science gaps impede use of no-take marine reserves. Trends Ecol. Evol. 20, 74-80 (2005).

5. Beger, M. et al. Incorporating asymmetric connectivity into spatial decision making for conservation. Cons. Lett. 3, 359-368 (2010).

6. Magris, R. A., Pressey, R. L., Weeks, R. \& Ban, N. C. Integrating connectivity and climate change into marine conservation planning. Biol. Cons. 170, 207-221 (2014).

7. Roberts, C. M. Connectivity and management of Caribbean coral reefs. Science 278, 1454-1457 (1997).

8. Cowen, R. K. \& Sponaugle, S. Larval dispersal and marine population connectivity. Annu. Rev. Mar. Sci. 1, 443-466 (2009).

9. Cowen, R. K., Paris, C. B. \& Srinivasan, A. Scaling of connectivity in marine populations. Science 311, 522-527 (2006).

10. Levin, L. A. Recent progress in understanding larval dispersal: new directions and digressions. Integr. Comp. Biol. 46, 282-297 (2006)

11. Pinsky, M. L., Palumbi, S. R., Andrefouet, S. \& Purkis, S. J. Open and closed seascapes: where does habitat patchiness create populations with high fractions of self-recruitment? Ecol. Applic. 22, 1257-1267 (2012).

12. Lett, C., Nguyen-Huu, T., Cuif, M., Saenz-Agudelo, P. \& Kaplan, D. M. Linking local retention, self-recruitment, and persistence in marine metapopulations. Ecology 96, 2236-2244 (2015).

13. Burgess, S. C. et al. Beyond connectivity: how empirical methods can quantify population persistence to improve marine protected area design. Ecol. Appl. 24, 257-270 (2014). 
14. Simpson, S. D., Harrison, H. B., Claereboudt, M. R. \& Planes, S. Longdistance dispersal via ocean currents connects clownfish populations throughout entire species range. PLoS ONE 9, e107610 (2014).

15. Jones, G. P. et al. Larval retention and connectivity among populations of corals and reef fishes: history, advances and challenges. Coral Reefs 28, 307-325 (2009).

16. Jones, G. P. in Ecology of Fishes on Coral Reefs: The Functioning of an Ecosystem in a Changing World (ed. Mora, C.) 16-27 (Cambridge Univ. Press, 2015).

17. Jones, G. P., Milicich, M. J., Emslie, M. J. \& Lunow, C. Self-recruitment in a coral reef fish population. Nature 402, 802-804 (1999).

18. Almany, G. R., Berumen, M. L., Thorrold, S. R., Planes, S. \& Jones, G. P. Local replenishment of coral reef fish populations in a marine reserve. Science 316, 742-744 (2007).

19. Jones, G. P., Thorrold, S. R. \& Planes, S. Coral reef fish larvae settle close to home. Curr. Biol. 15, 1314-1318 (2005).

20. Planes, S., Jones, G. P. \& Thorrold, S. R. Larval dispersal connects fish populations in a network of marine protected areas. Proc. Natl Acad. Sci. USA 106, 5693-5697 (2009).

21. Christie, M. R. et al. Larval connectivity in an effective network of marine protected areas. PLoS ONE 5, e15715 (2010).

22. Berumen, M. L. et al. Persistence of self-recruitment and patterns of larval connectivity in a marine protected area network. Ecol. Evol. 2, 444-452 (2012).

23. Saenz Agudelo, P., Jones, G. P., Thorrold, S. R. \& Planes, S. Connectivity dominates larval replenishment in a coastal coral reef fish metapopulation. Proc. R. Soc. B. 278, 2954-2961 (2010).

24. Harrison, H. B. et al. Larval export from marine reserves and the recruitment benefit for fish and fisheries. Curr. Biol. 22, 1023-1028 (2012).

25. D’Aloia, C. C. et al. Patterns, causes, and consequences of marine larval dispersal. Proc. Natl Acad. Sci. USA 112, 13940-19945 (2015).

26. Almany, G. R. et al. Dispersal of grouper larvae drives local resource sharing in a coral reef fishery. Curr. Biol. 23, 626-630 (2013).

27. Bonin, M. C. et al. The role of marine reserves in the replenishment of a locally impacted population of anemonefish on the Great Barrier Reef. Mol. Ecol. 25, 487-499 (2016).

28. Wabnitz, C., Taylor, M., Green, E \& Razak, T. From Ocean to Aquarium: The Global Trade in Marine Ornamental Species (UNEP-WCMC, 2003).

29. Jones, G. P., McCormick, M. I., Srinivasan, M. \& Eagle, J. V. Coral decline threatens fish biodiversity in marine reserves. Proc. Natl Acad. Sci. USA 101, $8251-8253$ (2004)

30. Green, A. et al. Designing a resilient network of marine protected areas for Kimbe Bay, Papua New Guinea. Oryx 43, 488-498 (2009).

31. Gerber, S., Chabrier, P. \& Kremer, A. Famoz: a software for parentage analysis using dominant, codominant and uniparentally inherited markers. Mol. Ecol. Notes 3, 479-481 (2003).

32. Hoegh-Guldberg, O. et al. Coral reefs under rapid climate change and ocean acidification. Science 318, 1737-1742 (2007)

33. Nanninga, G. B., Saenz-Agudelo, P., Zhan, P., Hoteit, I. \& Berumen, M. L. Not finding Nemo: limited reef-scale retention in a coral reef fish. Coral Reefs 34, 383-392 (2015).

34. Salles, O. C. et al. Coral reef populations can persist without immigration. Proc. R. Soc. B. 282, 2015131 (2015).

35. Williamson, D. H. et al. Large-scale, multidirectional larval connectivity among coral reef fish populations in the Great Barrier Reef Marine Park. Mol. Ecol. 25, 6039-6054 (2016)

36. Pinsky, M. L. et al. Marine dispersal scales are congruent over evolutionary and ecological time. Curr. Biol. 27, 1-6 (2017).

37. Steinberg, C. R., Choukroun, S. M., Slivkoff, M. M., Mahoney, M. V. \& Brinkman, R. M. Currents in the Bismarck Sea and Kimbe Bay, Papua New Guinea. TNC Pacific Islands Countries Report 6/06 (Australian Institute of Marine Science/The Nature Conservancy, 2006).

38. Lobel, P. S. \& Robinson, A. R. Transport and entrainment of fish larvae by ocean mesoscale eddies and currents. Deep-Sea Res. 33, 483-500 (1986).
39. Shulzitski, K., Sponaugle, S., Hauf, M., Walter, K. D. \& Cowen, R. K. Encounter with mesoscale eddies enhances survival in larval coral reef fishes. Proc. Natl Acad. Sci. USA 113, 6928-6933 (2016).

40. Gerlach, G., Atema, J., Kingsford, M. J., Black, K. P. \& Miller-Sims, V. Smelling home can prevent dispersal of reef fish larvae. Proc. Natl Acad. Sci. USA 104, 858-863 (2007).

41. Dixson, D. L. et al. Coral reef fish smell leaves to find island homes. Proc. R. Soc. B 275, 2831-2839 (2008).

42. Berumen, M. L. et al. Otolith geochemistry does not reflect dispersal history of clownfish larvae. Coral Reefs 29, 883-891 (2010).

43. Lester, S. E. et al. Biological effects within no-take reserves: a global synthesis Mar. Ecol. Prog. Ser. 384, 33-46 (2009).

44. Emslie, M. J. et al. Expectation and outcomes of reserve network performance following re-zoning of the Great Barrier Reef Park. Curr. Biol. 25, 983-992 (2015)

45. Cowen, R. K., Gawarkiewicz, G., Pineda, J., Thorrold, S. R. \& Werner, F. E. Population connectivity in marine systems: an overview. Oceanography 20, 14-21 (2009)

46. Kaplan, D. M., Botsford, L. W. \& Jorgensen, S. Dispersal-per-recruit: an efficient method for assessing sustainability in networks of marine reserves. Ecol. Appl. 16, 2248-2263 (2006).

47. Almany, G. R. Marine ecology: reserves are necessary, but not sufficient. Curr. Biol. 25, R328-R347 (1998).

48. Harrison, H. B., Saenz-Agudelo, P., Planes, S., Jones, G. P. \& Berumen, M. L. Relative accuracy of three common methods of parentage analysis in natural populations. Mol. Ecol. 22, 1158-1170 (2013).

49. McCormick, M. I. \& Choat, J. H. Estimating total abundance of a large temperate reef fish using visual strip-transects. Mar. Biol. 96, 469-478 (1987).

\section{Acknowledgements}

We thank the volunteers who dedicated long hours in the water collecting tissue samples: R. Brooker, S. Choukroun, P. Costello, J. Davies, D. Dixson, K. Furby, M. Giru, B. Grover J. Hill, N. Jones, K. McMahon, M. Noble, S. Noonan, N. Raventos Klein, M. Pinsky, J. Roberts, J. Smith, N. Tolou, M. Takahashi, P. Waldie and M. White; and the people of the villages on the shores of Kimbe Bay who welcomed us into their communities and supported this research: Kilu-Tamare, Lolobau, Tairobe and Vaiaku. This research would not have been possible without the support of the Walindi Plantation Resort, the skipper and crew of MV Febrina, Mahonia Na Dari Research and Conservation Centre, and The Nature Conservancy. This work was supported by Australian Research Council funding to G.P.J., the King Abdullah University of Science and Technology (baseline research funds to M.L.B. and a Special Partnership Collaborative Fellowship to M.L.B. and P.S.-A.) and NSF grants OCE0928442 and OCE1031256 to S.R.T.

\section{Author contributions}

G.R.A., M.L.B., G.P.J., S.P. and S.R.T designed the study. All authors contributed to field work and editing of the manuscript. M.B., H.B.H., S.P., M.A.P., P.S.-A. and S.P. conducted microsatellite DNA analyses. G.R.A. and M.B. developed the dispersal kernel model M.B., H.H. and S.R.T. created figures.

\section{Additional information}

Supplementary information is available for this paper.

Reprints and permissions information is available at www.nature.com/reprints.

Correspondence and requests for materials should be addressed to S.R.T.

How to cite this article: Almany, G. R. et al. Larval fish dispersal in a coral-reef seascape. Nat. Ecol. Evol. 1, 0148 (2017).

Publisher's note: Springer Nature remains neutral with regard to jurisdictional claims in published maps and institutional affiliations.

\section{Competing interests}

The authors declare no competing financial interests. 